\title{
Anti-cancer effects of Rhizoma Curcumae against doxorubicin-resistant breast cancer cells
}

\author{
Zhangfeng Zhong ${ }^{1,2+}$, Haibing $\mathrm{Yu}^{3 \dagger}$, Shengpeng Wang ${ }^{2}$, Yitao Wang ${ }^{2^{*}}$ and Liao Cui ${ }^{*^{*}}$
}

\begin{abstract}
Background: Chemotherapy is a primary approach in cancer treatment after routine surgery. However, chemoresistance tends to occur with chemotherapy in clinic, resulting in poor prognosis and recurrence. Nowadays, Chinese medicine may shed light on design of new therapeutic modes to overcome chemo-resistance. Although Rhizoma Curcumae possesses anti-cancer activities in various types of cancers, the effects and underlying mechanisms of its bioactive components against chemo-resistance are not clear. Therefore, the present study aims to explore the potential effects of Rhizoma Curcumae on doxorubicin-resistant breast cancer cells.

Methods: The expression and function of ABC transporters in doxorubicin-resistant MCF-7 breast cancer cells were measured by western blotting and flow cytometry. Cell viability was detected using MTT assay. The combination index was analyzed using the CalcuSyn program (Biosoft, Ferguson, MO), based on the Chou-Talalay method.

Results: In our present study, P-gp was overexpressed at protein level in doxorubicin-resistant MCF-7 cell line, but short of MRP1 and BCRP1. Essential oil of Rhizoma Curcumae and the main bioactive components were assessed on doxorubicin-resistant MCF-7 cell line. We found that the essential oil and furanodiene both display powerful inhibitory effects on cell viability, but neither of these is the specific inhibitor of $A B C$ transporters. Moreover, furanodiene fails to enhance the efficacy of doxorubicin to improve multidrug resistance.

Conclusion: Overall, our findings fill the gaps of the researches on chemo-resistance improvement of Rhizoma Curcumae and are also beneficial for Rhizoma Curcumae being developed as a promising natural product for cancer adjuvant therapy in the future.
\end{abstract}

Keywords: Rhizoma Curcumae, Multidrug resistance, Breast cancer, ABC transporters, Furanodiene

\section{Background}

Chemotherapy is regarded as one of adjuvant therapy after a routine surgery and being the primary approach for various cancer types [1-3]. However, many obstacles, including low efficacy and side effects, especially for chemo-resistance, still exist in cancer patients undergoing chemotherapy. There are a lot of strategies overcoming chemo-resistance, such as targeting ATP-binding cassette $(\mathrm{ABC})$ transporters $[4,5]$, inducing cell apoptosis

\footnotetext{
*Correspondence: ytwang@umac.mo; cuiliao@163.com

†Zhangfeng Zhong and Haibing Yu contributed equally to this work ${ }^{1}$ Guangdong Key Laboratory for Research and Development of Natural Drugs, Guangdong Medical University, Zhanjiang, Guangdong, China ${ }^{2}$ State Key Laboratory of Quality Research in Chinese Medicine, Institute of Chinese Medical Sciences, University of Macau, Macao, China Full list of author information is available at the end of the article
}

[6], inhibiting DNA repair [7], regulating metabolic reprogramming $[8,9]$, or applying combination therapy [10]. The role of $\mathrm{ABC}$ transporters is found to be closely related with chemo-resistance, thereby leading to poor prognosis and tumor recurrence in clinic [11]. Because of the expressions of $\mathrm{ABC}$ transporters, efflux pump decreases intracellular accumulation of drugs, then therapeutic concentrations of effective agents are reduced [12]. A thorough mechanisms of $\mathrm{ABC}$ transporters in chemo-resistance are still ongoing, and some typical proteins have been hot topics for a long time, including P-glycoprotein 1 (P-gp, MDR1, or ABCB1) [13], multidrug resistance-associated protein 1 (MRP1) [14], and ATP-binding cassette sub-family G member 2 (ABCG2 or BCRP) $[15,16]$. 
Overcoming chemo-resistance is a big challenge to chemotherapy. Natural products are rich sources of bioactive constitutes reversing cancer multidrug resistance, enhancing efficacy of anti-cancer agents, and decreasing side effects $[9,17,18]$. Currently, growing evidence show that Rhizoma Curcumae exhibits therapeutic value in overcoming chemo-resistance. The fractionated extracts of Rhizoma Curcumae improve the sensitivity of doxorubicin-resistant MCF-7 breast cancer cells to doxorubicin by blocking P-gp activity and down-regulating P-gp expression [19]. Furthermore, the fraction of $\mathrm{CH}_{2} \mathrm{Cl}_{2}$ extract is much more effective than that of EtOAc extract to restore the sensitivity of chemo-resistant MCF-7 cells to anti-neoplastic agents [20]. Meanwhile, several pure compounds (as shown in Fig. 1) isolated from Rhizoma Curcumae also have been reported to possess anti-cancer activities in multidrug resistant cancer cells. In detailly, curcumin inhibits viability of chemo-resistant breast cancer cells in an ER-independent manner and reverses multidrug resistance through $\mathrm{ABC}$ transporters [21]. $\beta$-elemene enhances cytotoxic effect of doxorubicin on doxorubicin-resistant MCF-7 breast cancer cells, which is related to increased doxorubicin accumulation and decreased Bcl-2 expression [22]. Germacrone reverses multidrug resistance through inducing cell apoptosis by down-regulation of $\mathrm{Bcl}-2$ and up-regulation of p53 and Bax. In addition, germacrone significantly reduces P-gp expression in multidrug resistant breast cancer cells [23].

Combination therapy is a treatment combining two or more therapeutic agents, aiming to improve disease-specific symptoms and overall survival. During and after cancer treatment, combination therapy potentially reduces chemo-resistance and provide therapeutic anti-cancer benefits simultaneously [24, 25]. Meanwhile, a variety of components derived from Chinese medicinal herbs are undergoing extensive researches of combination treatments in overcoming multidrug resistance to enhance efficacy [26, 27]. Therefore, it is urgent to develop an integrative approach to cancer care, when combination therapy meets Chinese medicinal herbs [28, 29]. To the best of our knowledge, there is no report of furanodiene or essential oil from Rhizoma Curcumae exhibiting anti-cancer effects in chemo-resistant cancer cells by means of $A B C$ transporters regulation. Therefore, our present study identified anti-cancer effects of those bioactive constituents from Rhizoma Curcumae initially and explored the related mechanisms in doxorubicinresistant MCF-7 breast cancer cells.

\section{Methods}

The Minimum Standards of Reporting Checklist contains details of the experimental design, and statistics, and resources used in this study (Additional file 1).

\section{Chemical and reagents}

The Roswell Park Memorial Institute-1640 (RPMI-1640) culture medium were purchased from Gibco (Maryland, USA). Fetal bovine serum (FBS), phosphate-buffered saline (PBS), penicillin-streptomycin (PS), and $0.25 \%(\mathrm{w} / \mathrm{v})$ trypsin/1 mM EDTA were obtained from<smiles>C=C[C@]1(C)CC[C@@H](C(=C)C)C[C@H]1C(=C)C</smiles> 
Invitrogen (Carlsbad, USA). 3-[4, 5-Dimethyl-2-thiazolyl]-2, 5-diphenyltetrazolium bromide (MTT) and Calcein AM were obtained from Molecular Probes (Eugene, USA). Doxorubicin (DOX) and Rhodamine 123 were supplied by Sigma-Aldrich (St. Louis, USA). Furanodiene and furanodienone were purchased from National Institutes for Food and Drug Control. Essential oil was obtained as our previous report [30]. Radioimmunoprecipitation assay (RIPA) lysis buffer and primary antibodies against P-gp, MRP1, and BCRP1 were obtained from Santa Cruz (Santa Cruz, USA). Primary antibodies against $\beta$-actin, as well as the secondary antibodies were purchased from Cell Signaling (Danvers, USA).

\section{Cell culture and drug treatment}

MCF-7 cell line was obtained from the ATCC (Manassas, USA) and was cultured as previously reported [31]. To induce doxorubicin-resistant cancer cell line, MCF-7 cells were cultured with RPMI1640 medium containing fetal bovine serum $(10 \%)$, penicillin $(100$ units per $\mathrm{mL})$, and streptomycin $(100 \mu \mathrm{g} / \mathrm{mL})$, at $37^{\circ} \mathrm{C}$ in a humidified atmosphere of $5 \% \mathrm{CO}_{2}$ in air. Doxorubicin-resistance was established by stepwise exposure to increased concentrations of doxorubicin as described previously [32]. The stock solutions of essential oil $(100 \mathrm{mg} / \mathrm{mL})$, furanodiene (100 mM), furanodienone (100 mM), and doxorubicin (2 and $100 \mathrm{mM}$ ) dissolved in DMSO were diluted to different concentrations as required.

\section{Cell viability assay}

Cell viability was performed using MTT assay as described previously [33]. Briefly, exponentially growing cells were seeded in 96-well plates at a density of $2 \times 10^{4} \%$ well and allowed to attach overnight. Following the required incubation period, cell viability was determined by adding MTT working solution $(100 \mu \mathrm{L} /$ well, $1 \mathrm{mg} /$ $\mathrm{mL}$ ). The absorbance values at $570 \mathrm{~nm}$ were recorded using SpectraMax M5 microplate reader (Molecular Devices, Silicon Valley, USA).

\section{Western blotting assay}

Western blotting assay was performed according to previous studies [34]. Briefly, exponentially growing cells were seeded in culture dish $(100 \mathrm{~mm})$ at a density of $2 \times 10^{6} /$ dish and allowed to attach overnight. After the required treatments, cells were harvested, and the total proteins were extracted with RIPA lysis buffer. Equal amounts of total proteins were separated by appropriate SDS-PAGE followed by transferring onto a PVDF membrane. After blocking with 5\% non-fat milk, the membrane was incubated with specific primary antibodies $(1: 1000 \mathrm{v} / \mathrm{v})$ and the corresponding second antibodies $(1: 2000 \mathrm{v} / \mathrm{v})$, respectively. The specific protein bands were visualized with an Amersham ${ }^{\mathrm{TM}} \mathrm{ECL}^{\mathrm{TM}}$ advanced western blotting detection kit (GE Healthcare Life Sciences, UK).

\section{P-gp expression assay}

P-gp expression was evaluated using the antibody of P-glycoprotein conjugated FITC (BD Biosciences, San Jose, USA) as described previously [35]. Cells were seeded into 6-well plates at a density of $2 \times 10^{5} /$ well, followed by the required drug treatments. The cells were harvested and incubated with $100 \mu \mathrm{L}$ of P-gp-FITC antibody dye-loading buffer at $37{ }^{\circ} \mathrm{C}$ for 30 min protected from light. The mean fluorescence intensity of FITC was detected using a flow cytometry (BD FACS Canto ${ }^{\mathrm{TM}}$, BD Biosciences, San Jose, USA). And the results were analyzed by FlowJo software (TreeStar, Ashland, OR, USA).

\section{P-gp function assay}

Rhodamine 123 and Calcein AM were applied to determine the activity of P-gp as described previously [35]. Cells were seeded into 6-well plates at a density of $2 \times 10^{5} /$ well, followed by the required drug treatments. $100 \mu \mathrm{L}$ of Rhodamine 123 or Calcein AM dye-loading solutions were added to each well and incubated at $37{ }^{\circ} \mathrm{C}$ for $30 \mathrm{~min}$ protected from light. The cells were harvested, and intracellular fluorescence was detected using a flow cytometry and analyzed by FlowJo software.

\section{Doxorubicin uptake assay}

Cells were seeded in 6-well plates at a density of $2 \times 10^{5}$ / well and were treated with different concentrations of test agents in the presence of doxorubicin. Following a 2-h incubation, the cells were washed and re-suspended in dye-free culture medium. The doxorubicin uptake was assessed using flow cytometry and the results were also analyzed by FlowJo software.

\section{Statistical analysis}

All data represent the mean of three independently performed experiments, plus or minus standard deviation or standard error of the mean. The significance of intergroup differences was evaluated by one-way ANOVA using the GraphPad Prism software (GraphPad Software, USA). Newman-Keuls multiple comparison tests were performed for post hoc pairwise comparisons. $p$-values less than 0.05 were considered as significant.

\section{Results}

Establishment and characterization of doxorubicin-resistant MCF-7 breast cancer cell line Doxorubicin-resistant MCF-7 breast cancer cell line was established by a stepwise exposure of MCF-7 cells to increasing concentrations of doxorubicin. Cell viability was tested by MTT assay after a 48 -h treatment of 
doxorubicin. Our results show that doxorubicin-resistant MCF-7 cells are resistant to doxorubicin with an IC50 value of $73.45 \mu \mathrm{M}$. And MCF-7 cells are sensitive to doxorubicin with an IC50 value of $2.87 \mu \mathrm{M}$ (Fig. 2a). The drug resistance index (RI) is 25.60, calculated by the ratio of IC50 of doxorubicin-resistant MCF-7 cells and IC50 of MCF-7 cells. A chemo-resistant model with RI of 3 or more is considered a successful establishment. Then $\mathrm{ABC}$ transporters proteins were detected by western blotting. Results show that P-gp expression of doxorubicin-resistant MCF-7 cells is different from that of MCF-7 cells despite of absence or presence of doxorubicin. However, the protein expression levels of MRP1 and BCRP1 are not apparent both in MCF-7 cell line and doxorubicin-resistant MCF-7 cell line, even in the presence of doxorubicin (Fig. 2b). Furthermore, flow cytometry results confirm that the P-gp expression level of doxorubicin-resistant MCF-7 cells is much higher than that of MCF-7 cells, even in the presence of doxorubicin (Fig. 2c).

\section{Effects of bioactive constituents of Rhizoma Curcumae on P-gp protein expression in doxorubicin-resistant MCF-7 breast cancer cells}

Protein expression was assessed with western blotting, accompanied by FITC-P-gp staining assays. Different concentrations of essential oil (E30, E60, and E120 are 30,60 , and $120 \mu \mathrm{g} / \mathrm{mL}$ of essential oil, respectively) or furanodiene (F25, F50, and F100 are 25, 50, and $100 \mu \mathrm{M}$ of furanodiene, respectively) do not show any inhibitory effects on P-gp expression, as shown in Fig. 3a, b. Meanwhile, FITC-P-gp staining assay using flow cytometry show that when compared with the red histogram of isotype control IgG, there are no any significant alterations in P-gp expression after treatment of essential oil or furanodiene at the indicated concentrations. That means

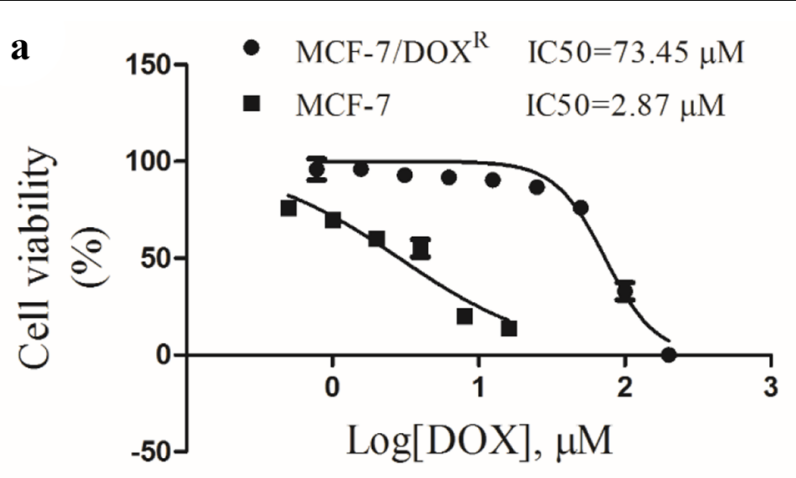

b

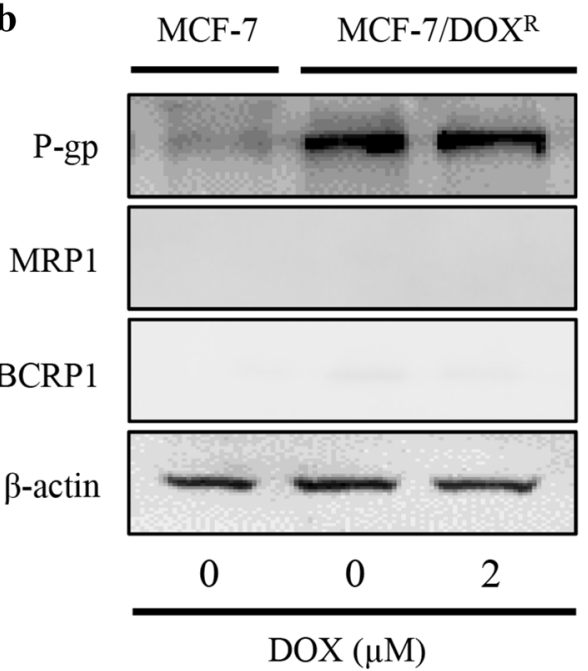

c

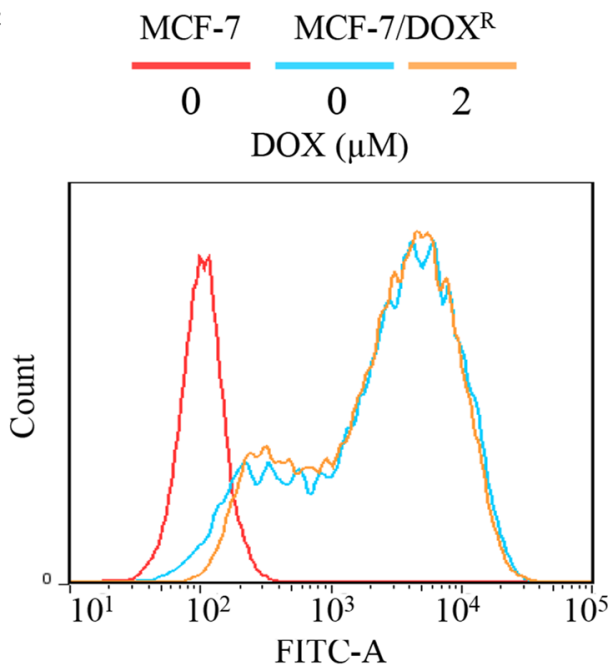

FITC-A

Fig. 2 Establishment and characterization of the doxorubicin (DOX)-resistant MCF-7 breast cancer cell line. Doxorubicin-resistant MCF-7 breast cancer cell line (MCF-7/DOX ${ }^{R}$ ) was established by a stepwise exposure of MCF-7 cells to increasing concentrations of doxorubicin (DOX). Cells were treated with DOX for $48 \mathrm{~h}$. a Cell viability was tested using MTT assay, represented by percentage of control. $\mathbf{b}$ Protein expression was evaluated using western blotting assay. c The expression alterations of P-gp were confirmed by FITC-P-gp antibody staining using flow cytometry 
$\mathbf{a}$

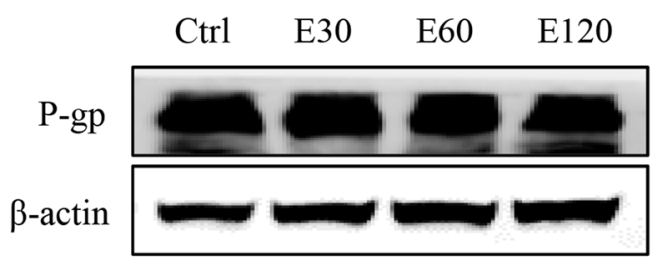

b

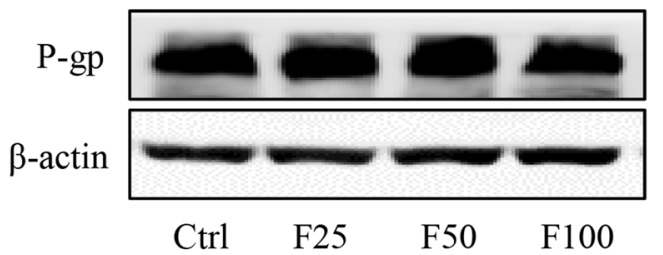

c

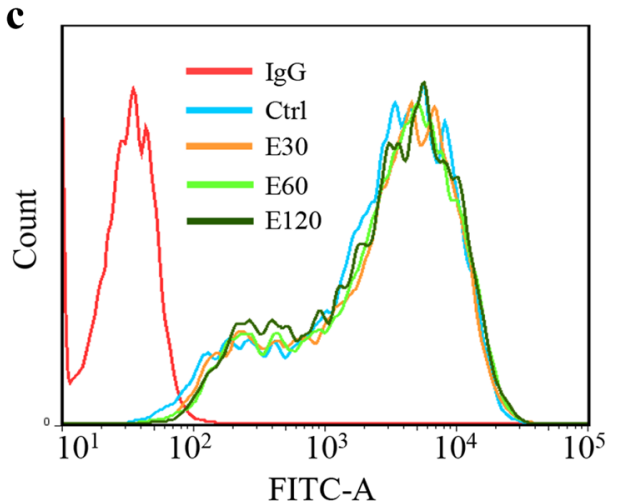

d

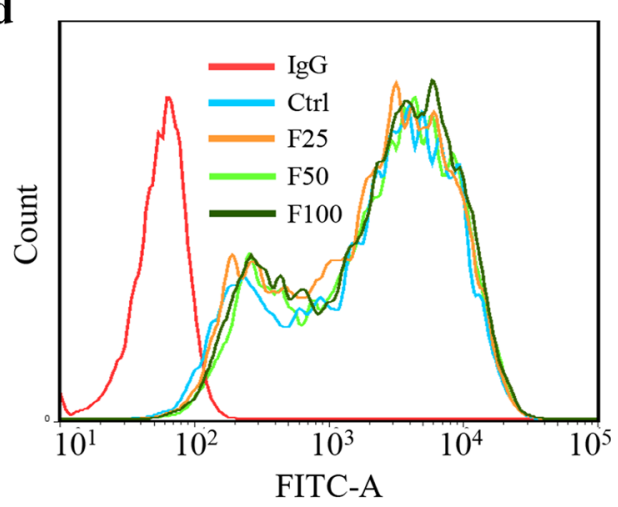

Fig. 3 Effects of bioactive constituents of Rhizoma Curcumae on P-gp protein expression in doxorubicin-resistant MCF-7 cells. Cells were treated with different concentrations of essential oil $(E ; \mu \mathrm{g} / \mathrm{mL})$ and furanodiene $(F ; \mu \mathrm{M})$ for $24 \mathrm{~h}$, compared with the control (Ctrl). $\mathbf{a}$, b Protein expression was evaluated using western blotting assay. $\mathbf{c}$, $\mathbf{d}$ Alterations of P-gp expression were confirmed by FITC-P-gp antibody staining using flow cytometry. Data were representative of at least three independent experiments

P-gp protein expression cannot be affected by essential oil or furanodiene in doxorubicin-resistant MCF-7 cells, as shown in Fig. 3c, d.

\section{Effects of bioactive constituents of Rhizoma Curcumae on P-gp function in doxorubicin-resistant MCF-7 breast cancer cells}

To further investigate influence of bioactive constituents on P-gp function, Rhodamine 123 and Calcein AM uptake assay were employed. After 1-h pre-treatment of essential oil or furanodiene, 30-min incubation with Rhodamine 123 or Calcein AM, fluorescence alterations were determined. Results show that $120 \mu \mathrm{g} / \mathrm{mL}$ of essential oil induces minor enhancements with mean fluorescence intensity (MFI) of 1359 and 2203, represented by P-gptransported Rhodamine 123 (Fig. 4a) and the intracellular Calcein (Fig. 4b). It indicates that essential oil exerts a slight inhibitory effect on P-gp function in doxorubicinresistant MCF-7 cells.

Regarding the potential effect of furanodiene on P-gp function, verapamil (VRP) and cyclosporine A (CYA) were used as the positive controls, both in Rhodamine 123 uptake assay and Calcein AM assay. In Rhodamine 123 uptake assay, compared with the remarkable increases (13.26-fold VRP and 37.28-fold CYA) induced by the positive controls, 1.47-fold increasement was observed after 100- $\mu \mathrm{M}$ furanodiene treatment (Fig. 5a, b). Similarly, in Calcein AM assay, compared with the remarkable increases (7.52-fold VRP and 7.16-fold CYA) induced by the positive controls, 1.77-fold increasement was observed after $100-\mu \mathrm{M}$ furanodiene treatment (Fig. 5c, d).

Collectively, essential oil and furanodiene, both exert a mild inhibitory effect on efflux activity of P-gp in doxorubicin-resistant MCF-7 cells. 


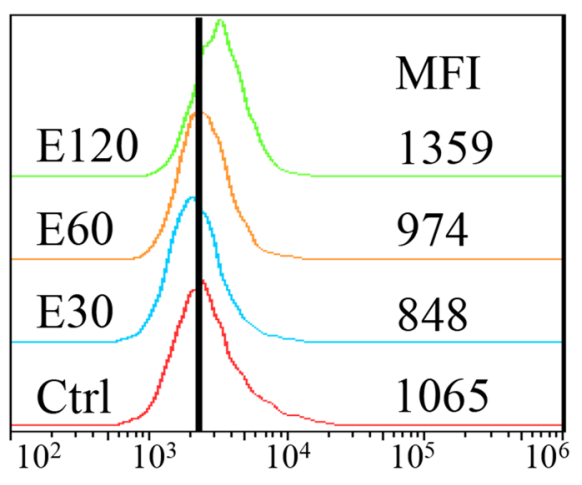

FITC-A

b

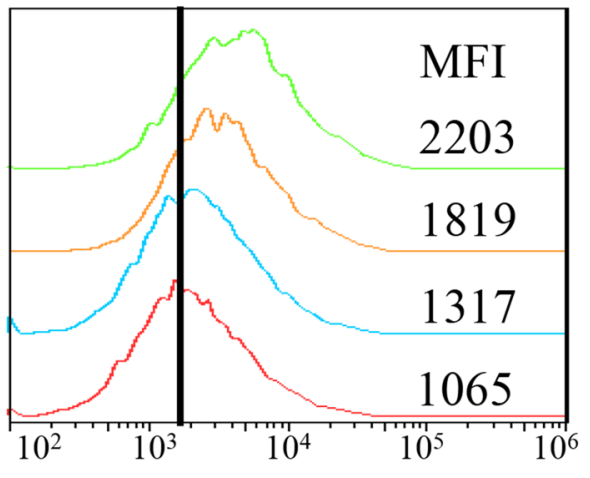

FITC-A

Fig. 4 Effect of essential oil on P-gp function in doxorubicin-resistant MCF-7 cells. P-gp function evaluation was performed by a 30-min incubation of a Rhodamine 123 and $\mathbf{b}$ Calcein AM using flow cytometry after 1-h treatment of essential oil ( $E ; \mu \mathrm{g} / \mathrm{mL})$. Ctrl stands for control. Data were representative mean fluorescence intensity (MFI) of at least three independent experiments

$\mathbf{a}$
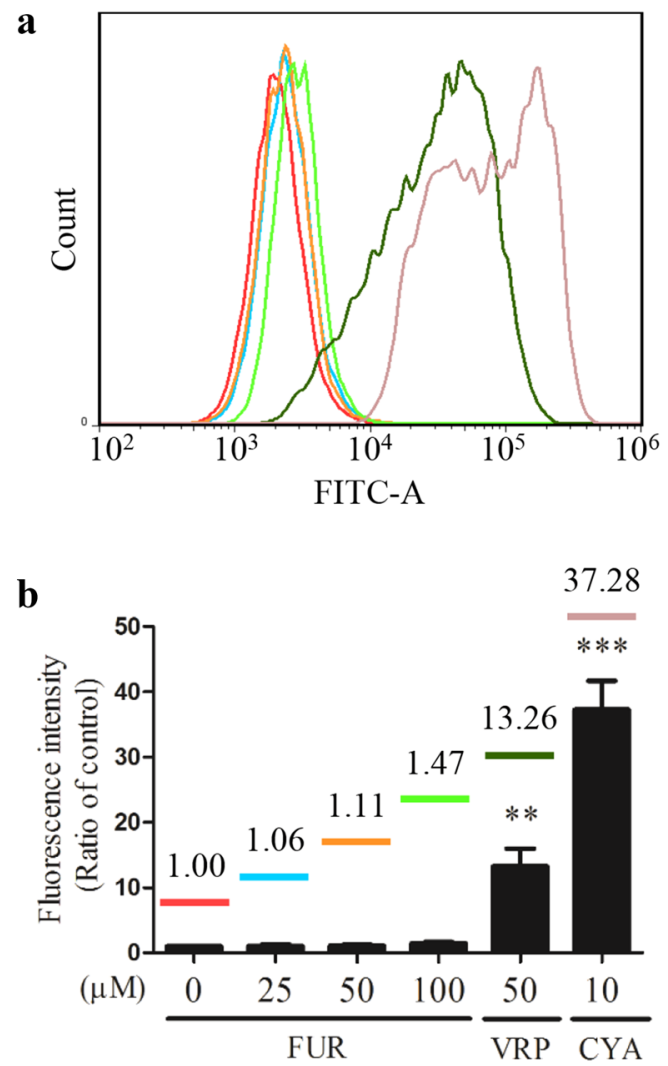

c
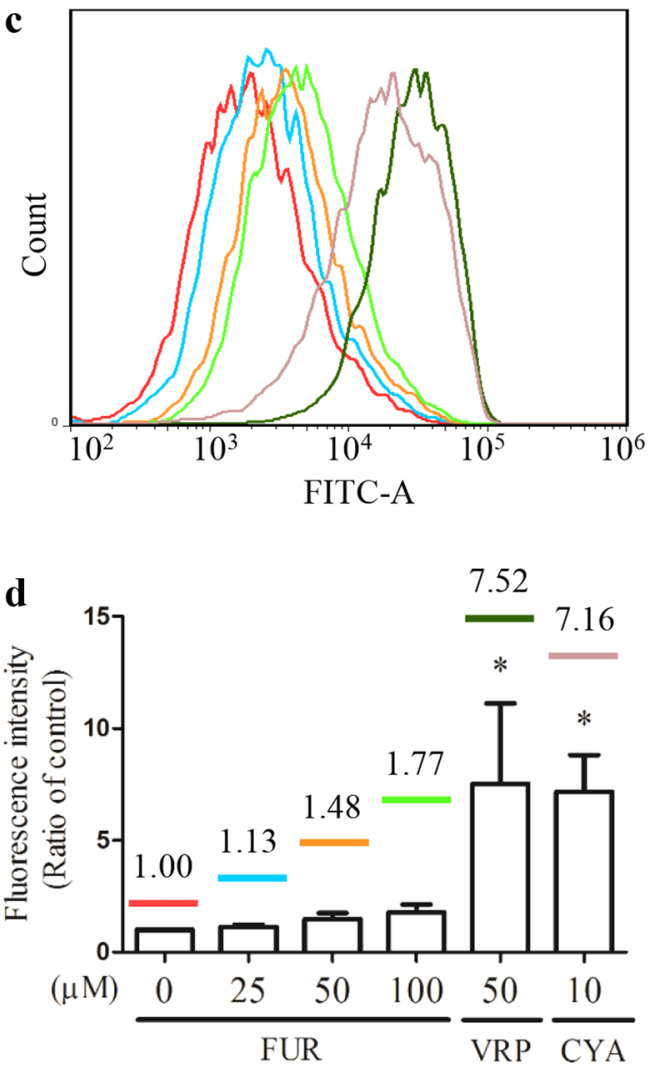

Fig. 5 Effect of furanodiene (FUR) on P-gp function in doxorubicin-resistant MCF-7 cells. P-gp function evaluation was performed by a 30-min incubation of Rhodamine 123 (a) and Calcein AM (c) using flow cytometry after 1-h treatments with furanodiene (FUR), verapamil (VRP), and cyclosporine A (CYA). The corresponding statistical result (b, d) was shown with VRP and CYA as positive controls. Data were expressed as mean \pm S.E.M. ${ }^{*} P<0.05,{ }^{* *} P<0.01$, and ${ }^{* * *} P<0.001$ vs. negative control 
The enhancement of furanodiene on doxorubicin uptake in doxorubicin-resistant MCF-7 breast cancer cells

Doxorubicin uptake assay was performed to further confirm the regulatory effect of furanodiene on P-gp function. Results show that the positive drug verapamil $(50 \mu \mathrm{M})$ can significantly increase doxorubicin uptake, represented by 19.06 -fold fluorescence intensity compared with control. Meanwhile, furanodiene at the indicated concentrations slightly increases doxorubicin uptake without significance (Fig. 6a, b), which indicates that furanodiene may not be a specific inhibitor of $A B C$ transporter protein as verapamil.

\section{Effects of bioactive constituents of Rhizoma Curcumae on viability of doxorubicin-resistant MCF-7 breast cancer cells}

To investigate the effect of Rhizoma Curcumae on viability of chemo-resistant cancer cells, doxorubicin-resistant MCF-7 cells were exposed to essential oil, furanodienone, and furanodiene. Cell viability was tested by MTT assay after a 48-h treatment. Results show that essential oil, furanodienone, and furanodiene display powerful inhibitory effects on viability of doxorubicin-resistant MCF-7 cells, with IC50 values of $76.98 \mu \mathrm{g} / \mathrm{mL}$ (Fig. 7a), $52.14 \mu \mathrm{M}$ (Fig. 7b), and 69.63 $\mu \mathrm{M}$ (Fig. 7c), respectively.

\section{Combined effects of furanodiene and doxorubicin on viability of doxorubicin-resistant MCF-7 breast cancer cells}

Combined effects of furanodiene and doxorubicin on the viability of doxorubicin-resistant MCF-7 cells were determined after $24 \mathrm{~h}$ of treatment. Figure $8 \mathrm{a}$, b show that the drug treatment alone (furanodiene or doxorubicin at concentrations of 25,50 , and $100 \mu \mathrm{M}$ ) dose-dependently inhibits the viability of doxorubicin-resistant MCF-7 cells. Doxorubicin $(2 \mu \mathrm{M})$ and furanodiene $(25 \mu \mathrm{M})$ are selected in subsequent experiments on account of nontoxic concentrations, as shown in Fig. 8a, b. However, there is no enhancement on sensitivity observed for furanodiene (Fig. 8a) or doxorubicin (Fig. 8b), no significance even at the highest concentration $(100 \mu \mathrm{M}$ of furanodiene or doxorubicin). To further assess the interaction of furanodiene and doxorubicin, cell viability results were analyzed using CalcuSyn program (Biosoft, Ferguson, MO), based on Chou-Talalay method. The combination index (CI) less than one is defined as synergism, while the CI greater than one is defined as antagonism. As shown in Fig. 8c, a synergistic inhibitory effect on the viability of doxorubicin-resistant MCF-7 cells is found when high concentrations of furanodiene combined with low concentrations of doxorubicin; On the contrary, drug antagonism occurs when low concentrations of furanodiene combined with high concentrations of doxorubicin.

\section{Discussion}

Growing evidence claim that Rhizoma Curcumae possesses anti-cancer activity mainly due largely in part to the essential oil and the bioactive components as well, containing curcumin, $\beta$-elemene, germacrone, furanodiene, furanodienone, and so on. However, the anti-cancer activities of Rhizoma Curcumae against chemo-resistant cancer cells are not clear yet. Therefore, we reviewed the anti-cancer effects of bioactive constituents of Rhizoma Curcumae against chemo-resistant cancer cells. Collectively, some reports demonstrated that Rhizoma
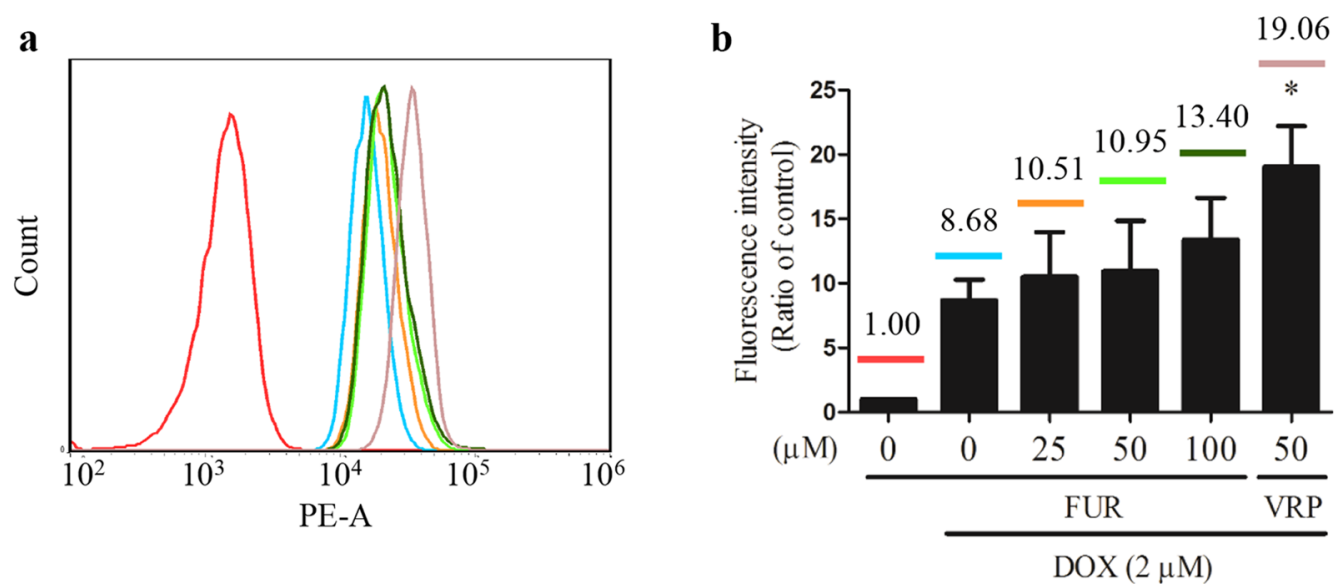

Fig. 6 Effect of furanodiene (FUR) on cellular uptake of doxorubicin in doxorubicin (DOX)-resistant MCF-7 cells. a After explosion to furanodiene (FUR) or verapamil (VRP) in the presence of doxorubicin (DOX) for $2 \mathrm{~h}$, doxorubicin uptake was analyzed using flow cytometry. b Statistical result of a. Data shown were expressed as mean \pm S.E.M. ${ }^{*} P<0.05$ vs. negative control 

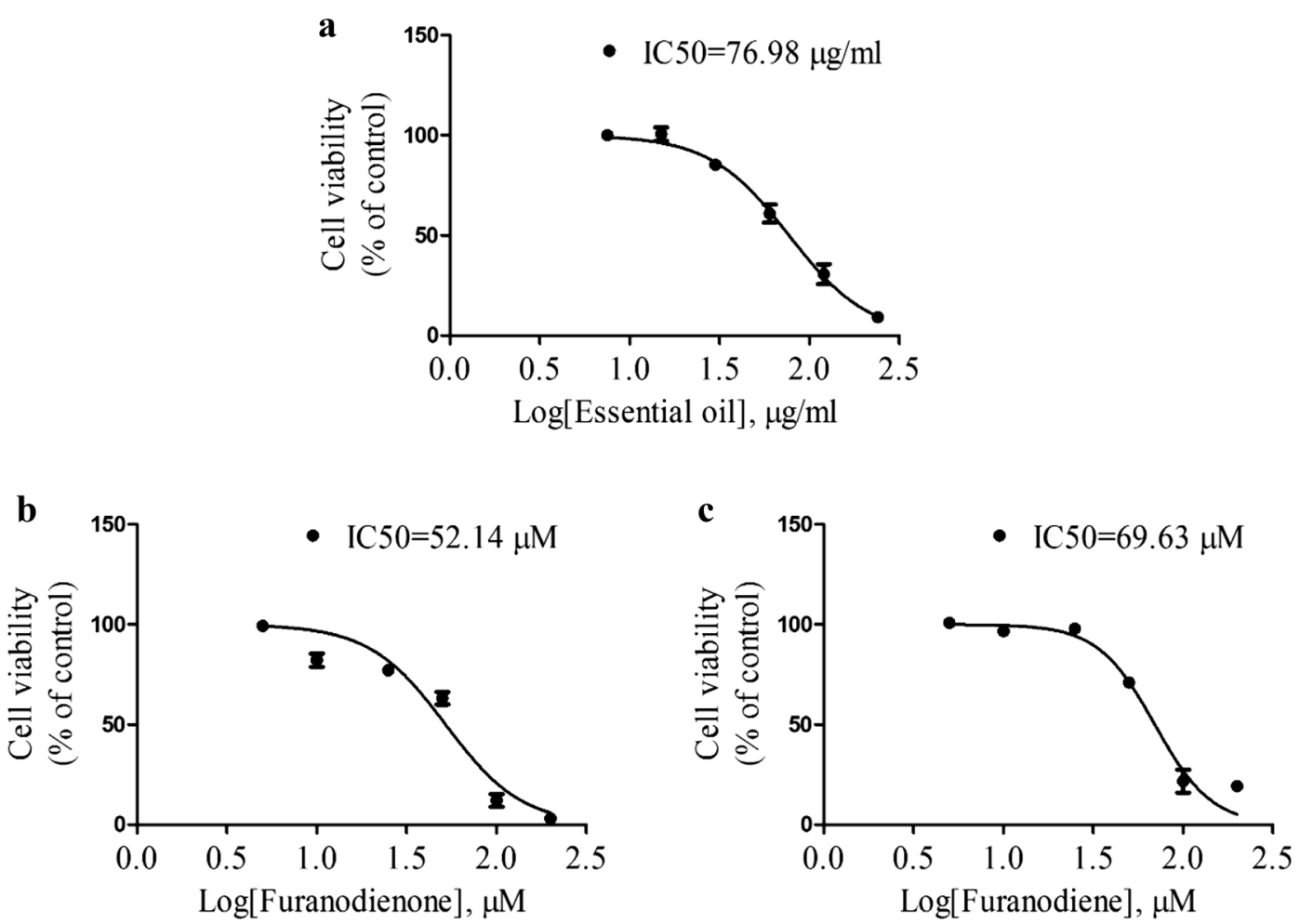

Fig. 7 Effects of bioactive constituents of Rhizoma Curcumae on viability of doxorubicin-resistant MCF-7 cells. Cells were treated with different concentrations of $\mathbf{a}$ essential oil, $\mathbf{b}$ furanodienone, and $\mathbf{c}$ furanodiene for $48 \mathrm{~h}$. Cell viability was tested using MTT assay. Data shown were expressed as mean \pm S.E.M

Curcumae extract [19], curcumin [36], $\beta$-elemene [37], and germacrone [23] exhibit anti-cancer activities in chemo-resistant cancer cells. However, to the best of our knowledge, there are still research gaps in the exact mechanisms of essential oil, furanodiene, and furanodienone on chemo-resistance. Therefore, our present study was designed to investigate the detailed anti-cancer effects of essential oil, furanodiene, and furanodienone against doxorubicin-resistant MCF-7 breast cancer cells.

Firstly, we identified whether these ingredients are specific inhibitors of $A B C$ transporters or not. Unexpectedly, essential oil and furanodiene cannot affect P-gp expression and slightly inhibit P-gp activity. Accordingly, the underlying mechanisms of Rhizoma Curcumae on chemo-resistance improvement may not be limited to the $\mathrm{ABC}$ transporters. Afterwards, our findings show that essential oil, furanodienone, and furanodiene display powerful inhibitory effects on viability of doxorubicin-resistant MCF-7 cells. The results clarified that these ingredients also have anti-cancer activities in chemo-resistant cancer cells. Meanwhile, furanodiene, the potential bioactive compound, was confirmed to induce extrinsic and intrinsic apoptosis through altering mitochondrial function via AMPK-dependent and
NF-kB-independent pathways in doxorubicin-resistant MCF-7 cells [32, 38].

According to our previous study, ER $\alpha$-negative MDAMB-231 cells are much more sensitive to furanodiene than ER $\alpha$-positive MCF-7 cells. Therefore, we concluded that furanodiene could significantly increase the efficacy of tamoxifen in ER $\alpha$-positive breast cancer cells [31]. Therefore, we make an inference from these results that furanodiene may enhance the efficacy of non-steroidal agents in ER $\alpha$-negative breast cancer cells, instead of in ER $\alpha$-positive breast cancer cells. Considering these findings mentioned above, we presumed that furanodiene could significantly enhance the efficacy of doxorubicin in ER $\alpha$-negative and ER $\alpha$-low expression breast cancer cells [39]. That is why we proposed the subsequent study evaluating the anti-breast cancer activities of furanodiene in combined with doxorubicin on doxorubicin-resistant breast cancer cells. Interestingly, it is actually observed that high concentrations of furanodiene combined low concentrations of doxorubicin exhibit synergistic inhibitory effects on the viability of doxorubicin-resistant MCF-7 cells, and low concentrations of furanodiene combined with high concentrations of doxorubicin exhibit antagonism. 
$\mathbf{a}$

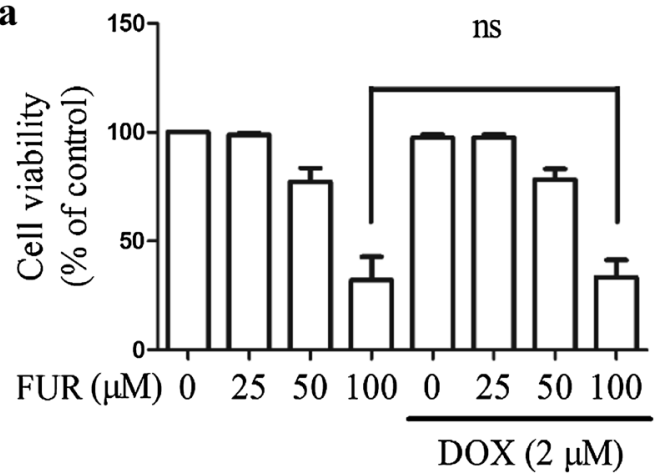

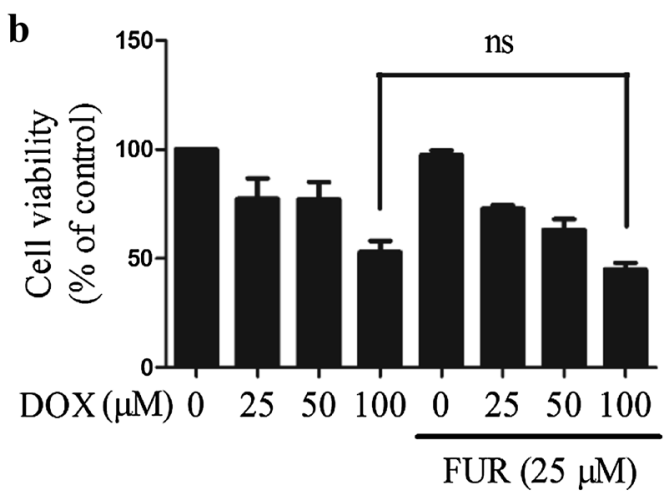

c

\begin{tabular}{cccc}
\hline FUR & \multicolumn{3}{c}{ Combination Index (CI) } \\
\cline { 2 - 4 }$(\mu \mathrm{M})$ & DOX $(25 \mu \mathrm{M})$ & DOX $(50 \mu \mathrm{M})$ & DOX $(100 \mu \mathrm{M})$ \\
\hline 12.5 & 1.083 & 1.665 & 1.831 \\
25 & 1.120 & 1.428 & 1.705 \\
50 & $0.900 *$ & 1.290 & 1.472 \\
100 & $0.464 *$ & $0.723 *$ & 1.123 \\
\hline
\end{tabular}

Fig. 8 Combined effects of furanodiene (FUR) and doxorubicin (DOX) on viability of DOX-resistant MCF-7 cells. a Cells were treated with different concentrations of furanodiene (FUR; $0-100 \mu \mathrm{M}$ ) in the presence or absence of doxorubicin (DOX; $2 \mu \mathrm{M})$ for $24 \mathrm{~h}$. $\mathbf{b}$ Cells were treated with different concentrations of DOX (0-100 $\mu \mathrm{M})$ in the presence or absence of FUR $(25 \mu \mathrm{M})$ for $24 \mathrm{~h}$. Cell viability was assessed using MTT assay. c The results were analyzed using CalcuSyn program (Biosoft), based on Chou-Talalay method. Data shown were expressed as mean \pm S.E.M. ns stands for not significant. *, synergism

\section{Conclusions}

Overall, even though essential oil and furanodiene are not the specific inhibitors of $A B C$ transporters, these ingredients still display powerful inhibitory effects on viability of doxorubicin-resistant breast cancer cells. The present study not only indicated Rhizoma Curcumae being a promising natural agent for cancer adjuvant therapy in the future, but also filled the gap of the researches on anti-cancer activities and corresponding mechanisms of Rhizoma Curcumae in chemo-resistant cancer cells.

\section{Additional file}

Additional file 1. Minimum Standards of Reporting Checklist.

\section{Abbreviations}

DOX: doxorubicin; FUR: furanodiene; VRP: verapamil; CYA: cyclosporine A; MTT: 3-[4, 5-Dimethyl-2-thiazolyl]-2, 5-diphenyltetrazolium bromide; Cl: combination index; $A B C$ transporters: ATP-binding cassette transporters; P-gp: P-glycoprotein 1; MRP1: multidrug resistance-associated protein 1; ABCG2: ATP-binding cassette sub-family G member 2; FBS: fetal bovine serum; PBS: phosphate-buffered saline; PS: penicillin-streptomycin; RIPA: radioimmunoprecipitation assay.

\section{Authors' contributions}

ZZ designed and performed the study. HY analyzed the data and revised the manuscript. SW participated in data analysis. YW and LC organized and supervised the study. All authors read and approved the final manuscript.

\section{Author details}

${ }^{1}$ Guangdong Key Laboratory for Research and Development of Natural Drugs, Guangdong Medical University, Zhanjiang, Guangdong, China. ${ }^{2}$ State Key Laboratory of Quality Research in Chinese Medicine, Institute of Chinese Medical Sciences, University of Macau, Macao, China. ${ }^{3}$ School of Public Health, Guangdong Medical University, Dongguan, Guangdong, China.

\section{Acknowledgements}

Not applicable.

Competing interests

The authors declare that they have no competing interests.

\section{Availability of data and materials}

Not applicable.

\section{Consent for publication}

Not applicable.

\section{Ethics approval and consent to participate} Not applicable.

\section{Funding}

This study was supported by the Macao Science and Technology Development Fund (071/2017/A2), the Research Fund of University of Macau (CPG2014-00012-ICMS), the China Postdoctoral Science Foundation Funded Project (2017M622811), the Natural Science Foundation of Guangdong 
Province (2018A030310226), and the Features Innovative Projects of General Colleges and Universities of Guangdong Province (4SG18126G).

\section{Publisher's Note}

Springer Nature remains neutral with regard to jurisdictional claims in published maps and institutional affiliations.

Received: 23 July 2018 Accepted: 22 August 2018

Published online: 29 August 2018

\section{References}

1. Kumagai K, Rouvelas I, Tsai JA, Mariosa D, Klevebro F, Lindblad M, et al. Meta-analysis of postoperative morbidity and perioperative mortality in patients receiving neoadjuvant chemotherapy or chemoradiotherapy for resectable oesophageal and gastro-oesophageal junctional cancers. Br J Surg. 2014;101(4):321-38.

2. Petrelli F, Borgonovo K, Cabiddu M, Lonati V, Barni S. Mortality, leukemic risk, and cardiovascular toxicity of adjuvant anthracycline and taxane chemotherapy in breast cancer: a meta-analysis. Breast Cancer Res Treat. 2012;135(2):335-46.

3. Ashraf N, Hoffe S, Kim R. Adjuvant treatment for gastric cancer: chemotherapy versus radiation. Oncologist. 2013;18(9):1013-21.

4. Schinkel AH, Jonker JW. Mammalian drug efflux transporters of the ATP binding cassette $(A B C)$ family: an overview. Adv Drug Deliv Rev. 2003;55(1):3-29.

5. Chen Z, Shi T, Zhang L, Zhu P, Deng M, Huang C, et al. Mammalian drug efflux transporters of the ATP binding cassette (ABC) family in multidrug resistance: a review of the past decade. Cancer Lett. 2016;370(1):153-64

6. Krishna R, Mayer LD. Multidrug resistance (MDR) in cancer. Mechanisms, reversal using modulators of MDR and the role of MDR modulators in influencing the pharmacokinetics of anticancer drugs. Eur J Pharm Sci. 2000;11(4):265-83.

7. Ding J, Miao ZH, Meng LH, Geng MY. Emerging cancer therapeutic opportunities target DNA-repair systems. Trends Pharmacol Sci. 2006;27(6):338-44.

8. Zhao Y, Butler EB, Tan M. Targeting cellular metabolism to improve cancer therapeutics. Cell Death Dis. 2013;4:e532.

9. Tan W, Zhong Z, Wang S, Liu H, Yu H, Tan R, et al. The typical metabolic modifiers conferring improvement in cancer resistance. Curr Med Chem. 2017:24(34):3698-710.

10. Al-Lazikani B, Banerji U, Workman P. Combinatorial drug therapy for cancer in the post-genomic era. Nat Biotechnol. 2012;30(7):679-92.

11. Lage $H$. ABC-transporters: implications on drug resistance from microorganisms to human cancers. Int J Antimicrob Agents. 2003;22(3):188-99.

12. Borowski E, Bontemps-Gracz MM, Piwkowska A. Strategies for overcoming $A B C$-transporters-mediated multidrug resistance (MDR) of tumor cells. Acta Biochim Pol. 2005;52(3):609-27.

13. Thomas $\mathrm{H}$, Coley HM. Overcoming multidrug resistance in cancer: an update on the clinical strategy of inhibiting p-glycoprotein. Cancer Control. 2003;10(2):159-65.

14. Liang Z, Wu H, Xia J, Li Y, Zhang Y, Huang K, et al. Involvement of miR-326 in chemotherapy resistance of breast cancer through modulating expression of multidrug resistance-associated protein 1. Biochem Pharmacol. 2010;79(6):817-24.

15. Bisson C, Adams NBP, Stevenson B, Brindley AA, Polyviou D, Bibby TS, et al. The molecular basis of phosphite and hypophosphite recognition by ABC-transporters. Nat Commun. 2017;8(1):1746.

16. Natarajan $K, X i e Y$, Baer MR, Ross DD. Role of breast cancer resistance protein (BCRP/ABCG2) in cancer drug resistance. Biochem Pharmacol. 2012;83(8):1084-103.

17. Cao YJ, Pu ZJ, Tang YP, Shen J, Chen YY, Kang A, et al. Advances in bioactive constituents, pharmacology and clinical applications of rhubarb. Chin Med. 2017;12:36

18. Zhang Y, Liang Y, He C. Anticancer activities and mechanisms of heatclearing and detoxicating traditional Chinese herbal medicine. Chin Med. 2017;12:20.
19. Yang L, Wei DD, Chen Z, Wang JS, Kong LY. Reversal of multidrug resistance in human breast cancer cells by Curcuma wenyujin and Chrysanthemum indicum. Phytomedicine. 2011;18(8-9):710-8.

20. Yang L, Wei DD, Chen Z, Wang JS, Kong LY. Reversal effects of traditional Chinese herbs on multidrug resistance in cancer cells. Nat Prod Res. 2011;25(19):1885-9.

21. Labbozzetta M, Notarbartolo M, Poma P, Maurici A, Inguglia L, Marchetti $P$, et al. Curcumin as a possible lead compound against hormoneindependent, multidrug-resistant breast cancer. Ann N Y Acad Sci. 2009;1155:278-83.

22. Hu J, Jin W, Yang PM. Reversal of resistance to adriamycin in human breast cancer cell line MCF-7/ADM by $\beta$-elemene. Zhonghua Zhong Liu Za Zhi. 2004:26(5):268-70.

23. Xie XH, Zhao H, Hu YY, Gu XD. Germacrone reverses Adriamycin resistance through cell apoptosis in multidrug-resistant breast cancer cells. Exp Ther Med. 2014;8(5):1611-5.

24. Bayat Mokhtari R, Homayouni TS, Baluch N, Morgatskaya E, Kumar S, Das $B$, et al. Combination therapy in combating cancer. Oncotarget. 2017;8(23):38022-43

25. Rationalizing combination therapies. Nat Med. 2017;23(10):1113.

26. Wang S, Wang L, Shi Z, Zhong Z, Chen M, Wang Y. Evodiamine synergizes with doxorubicin in the treatment of chemoresistant human breast cancer without inhibiting P-glycoprotein. PLoS ONE. 2014;9(5):e97512.

27. Zhao Y, Yang A, Tu P, Hu Z. Anti-tumor effects of the American cockroach, Periplaneta americana. Chin Med. 2017;12:26

28. Wang Z, Qi F, Cui Y, Zhao L, Sun X, Tang W, et al. An update on Chinese herbal medicines as adjuvant treatment of anticancer therapeutics. Biosci Trends. 2018;12(3):220-39.

29. Weerapreeyakul N, Machana S, Barusrux S. Synergistic effects of melphalan and Pinus kesiya Royle ex Gordon (Simaosong) extracts on apoptosis induction in human cancer cells. Chin Med. 2016;11:29.

30. Yang FQ, Li SP, Zhao J, Lao SC, Wang YT. Optimization of GC-MS conditions based on resolution and stability of analytes for simultaneous determination of nine sesquiterpenoids in three species of Curcuma rhizomes. J Pharm Biomed Anal. 2007;43(1):73-82.

31. Zhong ZF, Li YB, Wang SP, Tan W, Chen XP, Chen MW, et al. Furanodiene enhances tamoxifen-induced growth inhibitory activity of ERa-positive breast cancer cells in a PPARy independent manner. J Cell Biochem. 2012;113(8):2643-51.

32. Zhong ZF, Tan W, Qiang WW, Scofield VL, Tian K, Wang CM, et al. Furanodiene alters mitochondrial function in doxorubicin-resistant MCF-7 human breast cancer cells in an AMPK-dependent manner. Mol BioSyst. 2016;12(5):1626-37

33. Pauzi AZ, Yeap SK, Abu N, Lim KL, Omar AR, Aziz SA, et al. Combination of cisplatin and bromelain exerts synergistic cytotoxic effects against breast cancer cell line MDA-MB-231 in vitro. Chin Med. 2016;11:46.

34. Lai IC, Lai GM, Chow JM, Lee HL, Yeh CF, Li CH, et al. Active fraction (HS7) from Taiwanofungus camphoratus inhibits AKT-mTOR, ERK and STAT3 pathways and induces CDK inhibitors in CL1-0 human lung cancer cells. Chin Med. 2017;12:33

35. Zhong ZF, Tan W, Wang SP, Qiang WA, Wang YT. Anti-proliferative activity and cell cycle arrest induced by evodiamine on paclitaxel-sensitive and -resistant human ovarian cancer cells. Sci Rep. 2015:5:16415.

36. Anuchapreeda S, Leechanachai P, Smith MM, Ambudkar SV, Limtrakul PN. Modulation of P-glycoprotein expression and function by curcumin in multidrug-resistant human KB cells. Biochem Pharmacol. 2002;64(4):573-82

37. Xu HB, Li L, Fu J, Mao XP, Xu LZ. Reversion of multidrug resistance in a chemoresistant human breast cancer cell line by $\beta$-elemene. Pharmacology. 2012;89(5-6):303-12

38. Zhong ZF, Yu HB, Wang CM, Qiang WA, Wang SP, Zhang JM, et al. Furanodiene induces extrinsic and intrinsic apoptosis in doxorubicin-resistant MCF-7 breast cancer cells via NF-KB-independent mechanism. Front Pharmacol. 2017:8:648.

39. Zhong ZF, Qiang WA, Wang CM, Tan W, Wang YT. Furanodiene enhances the anti-cancer effects of doxorubicin on ERa-negative breast cancer cells in vitro. Eur J Pharmacol. 2016;774:10-9. 